\title{
Evaluation of the Chemopreventive Activity of Grape Skin Extract Using Medium-term Oral Carcinogenesis Assay Induced by 4-Nitroquinoline 1-Oxide
}

\author{
CAROLINA FOOT GOMES DE MOURA ${ }^{1}$, GLAUCIA RESENDE SOARES ${ }^{1}$, \\ FLAVIA ANDRESSA PIDONE RIBEIRO ${ }^{1}$, MARCELO JOSE DIAS SILVA ${ }^{2}$, \\ WAGNER VILEGAS ${ }^{2}$, ALINE BOVETO SANTAMARINA ${ }^{1}$, \\ LUCIANA PELLEGRINI PISANI $^{1}$, DEBORA ESTADELLA ${ }^{1}$ and DANIEL ARAKI RIBEIRO ${ }^{1}$ \\ ${ }^{1}$ Department of Biosciences, Federal University of São Paulo, UNIFESP, Santos, Brazil; \\ ${ }^{2}$ São Paulo State University, UNESP, São Vicente, Brazil
}

\begin{abstract}
Background/Aim: The aim of this study was to evaluate the chemoprotective potential of grape skin extract following rat tongue carcinogenesis induced by 4nitroquinoline 1-oxide (4NQO). Materials and Methods: Male Wistar rats were distributed into four groups $(n=5$, per group): Control Group: free access to commercial diet and drinking water for 12 weeks; $4 N Q O$ Group: received $4 N Q O$ diluted in drinking water daily, for 12 weeks; Grape Skin Extract Group: free access to water and received grape skin extract incorporated with diet for 12 weeks; $4 N Q O+$ Grape Skin Extract Group: received $4 N Q O$ in drinking water daily and grape extract incorporated with diet for 12 weeks. Results: Animals treated with grape skin extract revealed a significant reduction in epithelial dysplasia. Also, 8-hydroxy$2^{\prime}$-deoxyguanosine (8-OHdG) and ki-67 immunoexpression was reduced in animals treated with grape skin extract. Western blot analysis showed a significant decrease of $p$ $N F K B$ p50 and MyD88 protein expression in the groups treated with grape skin extract. Copper-zinc superoxide dismutase, manganese superoxide dismutase, and catalase gene expression did not present any statistically significant differences $(p>0.05)$. Conclusion: Grape skin extract displayed chemopreventive activity in oral carcinogenesis assays as depicted by its antioxidant, anti-proliferative and anti-inflammatory properties.
\end{abstract}

Correspondence to: Daniel A. Ribeiro, DDS, Ph.D., Departamento de Biociências, Universidade Federal de São Paulo - UNIFESP, Av. Ana Costa, 95, Vila Mathias, Santos - SP, 11060-001, Brazil. Tel: +55 1338783774, e-mail: daribeiro@unifesp.br

Key Words: Oral cancer, rat, 4-nitroquinoline 1-oxide, proliferation, antioxidant activity, toll like signaling pathway.
Cancer was responsible for more than 8 million deaths worldwide in 2012 (1). Head and neck cancers represent $10 \%$ of all malignant tumors, and $40 \%$ of them are in the oral cavity (2). The major factors associated with an increased risk for developing oral cancer are smoking and the consumption of alcoholic beverages (3).

4-nitroquinoline 1-oxide (4NQO) is a well-established synthetic carcinogen derived from quinoline. Administration of $4 \mathrm{NQO}$ dissolved in water, is a simple and suitable method to initiate and promote oral carcinogenesis (4). The experimental model, develops hyperplastic, dysplastic and squamous cell carcinoma in the oral cavity, very similar to that observed in humans (5). For this reason, our research group has systematically used this model for studying oral cancer pathogenesis (6-9).

Grape is rich in phytochemical compounds, such as polyphenols. This fruit, as well as its derivatives, contain high levels of resveratrol, flavonoids, phenolic acids, and anthocyanins, and displays anti-mutagenic, anti-genotoxic and antioxidant properties in multiple organs and tissues (10). Although there are certain studies investigating the biological action of grape extracts and related compounds against oral cancer cells in vivo and in vitro $(11,12)$, no studies have examined whether grape skin extract has any chemopreventive activity following rat tongue carcinogenesis induced by $4 \mathrm{NQO}$.

The aim of this study was to evaluate the chemopreventive activity of grape skin extract following induction of rat tongue carcinogenesis by $4 \mathrm{NQO}$.

\section{Materials and Methods}

Animals and experimental design This study was approved by the Animal Committee of Federal University of São Paulo, UNIFESP (number 5732270515). Male Wistar rats (8-weeks-old, 
Table I. Compounds identified in the grape skin extract.

\begin{tabular}{llcc}
\hline & Compound & {$[\mathrm{M}+\mathrm{H}]^{-/[\mathrm{M}+\mathrm{H}]^{+}}$} & MS/MS ions \\
\hline 1 & Coumaric acid & 163 & 119 \\
2 & Gallic acid & 169 & 125 \\
3 & Caffeic acid & 179 & 135 \\
4 & Ferulic acid & 193 & $178,149,134$ \\
5 & Resveratrol & 227 & 185,143 \\
6 & Cyanidin & $287[\mathrm{M}+\mathrm{H}]^{+}$ & $165,241,213$ \\
7 & Catechin & 289 & 245 \\
8 & Quercetin & 301 & $301,179,151,107$ \\
9 & Delphinidin & $303[\mathrm{M}+\mathrm{H}]^{+}$ & $257,229,285,165,137$ \\
10 & Caftaric acid & 311 & 149 \\
11 & Coumaric-O-hexoside & 325 & 163,119 \\
12 & Hydroxycinnamic acid derivative & 421 & 287 \\
13 & Cyanidin-3-O-glucoside & $449[\mathrm{M}+\mathrm{H}]^{+}$ & 331 \\
14 & Malvidin-3-O-glucoside & $493[\mathrm{M}+\mathrm{H}]^{+}$ & \\
15 & Procyanidin dimer type B & 577 & $425,451,407,559,289$ \\
16 & Cyanidin-O-dihexoside & $611[\mathrm{M}+\mathrm{H}]^{+}$ & 303,465 \\
17 & Procyanidin trimer type B & 865 & $695,739,577,407,287$ \\
\hline
\end{tabular}

weighing approximately $250 \mathrm{~g}$ ) were purchased from the Development Center of Experimental Models for Medicine and Biology (CEDEME), Federal University of São Paulo, SP, Brazil. They were maintained under controlled conditions of temperature $\left(24 \pm 2^{\circ} \mathrm{C}\right)$, and light-dark periods of $12 \mathrm{~h}$. The animals were separated into four groups ( $\mathrm{n}=5$, per group), as follows: Control Group: free access to commercial diet (Nuvital ${ }^{\mathrm{TM}}$, PR, Brazil) and water for 12 weeks; 4NQO Group: received 4NQO diluted in drinking water daily, and commercial diet (Nuvital ${ }^{\mathrm{TM}}, \mathrm{PR}$, Brazil) for 12 weeks; Grape Skin Extract Group: free access to water and received grape skin extract $(2.5 \mathrm{mg} / \mathrm{kg})($ Christian Hansen $^{\mathrm{TM}}$, SP, Brazil) incorporated with diet for 12 weeks; 4NQO + Grape Skin Extract Group: received 4NQO in drinking water daily and grape extract $(2.5 \mathrm{mg} / \mathrm{kg})\left(\right.$ Christian Hansen ${ }^{\mathrm{TM}}$, SP, Brazil) incorporated with diet for 12 weeks. After completing the experimental period (12 weeks), all animals were euthanatized with inhalational of the anesthetic halothane (Tanohalo $^{\mathrm{TM}}$, Cristáliaa $^{\mathrm{TM}}$, Brazil).

The administration of 4NQO (Sigma Aldrich, St. Louis, MO, USA) was carried out at a dose of $50 \mathrm{ppm}$, diluted in drinking water and offered during the experimental period (12 weeks) as described elsewhere $(9,12)$. The daily dose offered was calculated in order to provide the equivalent dose to humans (i.e. $2 \mathrm{~g}$ polyphenols/day) based on the rat metabolism. This dosage is in accordance with that recommended by the American Dietetic Association and demonstrated in previous studies using polyphenols from grape (13).

Characterization of grape skin extract by electrospray ionization mass spectrometry (ESI-MSn). ESI-MSn data were obtained in the negative and positive ion mode with a Fleet LCQ Plus ${ }^{\mathrm{TM}}$ ion-trap instrument from Thermo Scientific ${ }^{\mathrm{TM}}$ (Karlsruhe, Germany). Data were acquired in MS1 and MSn scanning modes ranging from 100 to 2000. Xcalibur 2.2 software (Thermo Scientific ${ }^{\mathrm{TM}}$, Karlsruhe, Germany) was used for data analysis. The degree of confidence for this method was $99 \%$.
Histopathological analysis. The histopathological analysis of the tongue sections was performed following hematoxylin and eosin staining by light microscope, and scores were applied according to the intensity/severity of the lesions observed in each animal: no histopathological changes (score 0), hyperplasia (score 1), dysplasia (score 2), and squamous cell carcinoma (score 3 ).

Immunohistochemistry analysis. Immunohistochemistry for 8-OHdG and ki-67 was performed using an avidin-biotin complex protocol (12). A total of five sections per animal at $40 \times$ magnification were evaluated in "hot spot" areas, i.e. hyperplasia, dysplasia and carcinoma. These values were used as labeling indices.

Western blotting. Western blotting was conducted, as described by Yujra et al. (14). The antibody against pNFkBp50 (sc-101744) was purchased from Santa Cruz Biotechnology ${ }^{\mathrm{TM}}$, Inc. (Santa Cruz, CA, USA). The antibodies against MyD88 (ab2064), TRAF6 (ab33915) and GADPH (ab9484) were obtained from ABCAM ${ }^{\mathrm{TM}}$ (Cambridge, UK). The intensities of each band sample were quantified by Image $^{\mathrm{TM}}$ software (Image ${ }^{\mathrm{TM}}$, National Institute of Health, MD, USA). All numerical values were normalized using GADPH levels in the respective membrane.

Real-Time PCR. Gene expression of Copper-Zinc Superoxide Dismutase (CuZn-SOD), Manganese Superoxide Dismutase (MnSOD), and catalase genes were analyzed by real-time polymerase chain reaction (real-time PCR). The methodology was conducted according to de Ribeiro et al. (9).

Statistical analysis. Kruskal-Wallis non-parametric test followed by Dunn's post-test was applied to histopathological analysis, immunohistochemistry and western blotting data. One-way analysis of variance (one way - ANOVA) followed by Tukey's multiple comparisons post-hoc was used to analyze the real time-PCR data. The statistical analysis was performed using Graph Pad Prism ${ }^{\mathrm{TM}} 6.0$ program. $p<0.05$ was considered statistically significant. 
Table II. Histopathological analysis following induction of rat tongue carcinogenesis and treatment with grape skin extract.

\begin{tabular}{lcccc}
\hline & \multicolumn{3}{c}{ Scores } \\
\cline { 2 - 5 } & Score 0 & Score 1 & Score 2 & Score 3 \\
\hline Control Group & 5 & 0 & 0 & 0 \\
4NQO Group* & 0 & 0 & 2 & 3 \\
Grape Skin Extract Group & 5 & 0 & 3 & 0 \\
4NQO + Grape Skin Extract Group** & 0 & 2 & 3 & 0 \\
\hline
\end{tabular}

Score 0: No histopathological changes; score 1: epithelial hyperplasia; score 2: epithelial dysplasia; score 3: squamous cell carcinoma. ${ }^{*} p<0.05$ compared to Control Group; ${ }^{*} p<0.05$ compared to 4 NQO Group.

\section{Results}

Grape skin extract characterization/chemical analysis. A total of 17 constituents were identified with collision energy ramp from 25 to $30 \%$ and comparisons with fragmentation profiles (Table I). Several polyphenolic compounds were identified including: coumaric acid, gallic acid, caffeic acid, ferulic acid, resveratrol, cyanidin, catechin, quercetin, delphinidin, caftaric acid, coumaric-O-hexoside, hydroxycinnamic acid derivative, cyanidin-3-O-glucoside, malvidin-3-O-glucoside, procyanidin dimer type B, cyanidin-O-dihexoside and procyanidin trimer type B.

Histopathological analysis. The animals belonging to the control group or to the group that received a diet supplemented with grape skin extract had no alterations in the tongue epithelium. Among the animals submitted to the induction of the oral carcinogenesis, multiple foci of epithelial dysplasia were observed (4NQO Group). Three animals belonging to this group also presented carcinoma in the tongue epithelium. When animals were treated with grape skin extract, there was a reduction in the total number of animals that presented dysplasia. However, hyperplasia was present. The numerical results are presented in Table II.

Immunohistochemical analysis. Positive ki-67 staining was detected by immunohistochemistry as a brown color in the nucleus of oral keratinocytes. Weak nuclear immunoexpression was observed in animals of the control and grape skin extract groups. When animals were exposed to 4NQO, cell proliferation was highly elevated in the basal cells of the tongue epithelium. Conversely, animals that received 4NQO in drinking water and treated with grape skin extract simultaneously, displayed a significant reduction $(p<0.05)$ in the level of ki-67 immunopositive cells (Figure 1A).

Positive granular 8-OHdG staining was detected either in the nucleus or in the cytoplasm of epithelial cells. In the control group, weak 8-OHdG immunoreactivity was found in all animals. The same occurred in animals treated with grape skin extract only. Treatment with 4NQO induced strong cytoplasmic $8-\mathrm{OHdG}$ immunostaining in rat tongue cells compared to control $(p<0.05)$. However, a decrease in 8 -OHdG immunoexpression $(p<0.05)$ was observed in animals that received grape skin extract (Figure 1B).

Real time PCR. The analysis showed that the expression of $\mathrm{CuZn-SOD}$ was higher $(p<0.05)$ in animals exposed to the carcinogen compared to control group. Grape skin extract did not alter CuZn-SOD gene expression. Expression of MnSOD and catalase genes was not altered by 4NQO or by grape skin extract (Figure 2).

Western Blotting. Western blotting analysis showed that the expression levels of p-NFKB p50 and MyD88 proteins were decreased in the groups treated with grape skin extract group, that received $4 \mathrm{NQO}$ or not, compared to their respective controls $(p<0.05)$. Expression of TRAF-6 did not show statistically significant differences $(p>0.05)$ among groups (Figure 3 ).

\section{Discussion}

Recently, our research group investigated the therapeutic efficacy of natural products under different experimental conditions and paradigms, such as colitis, non-alcoholic steatosis and cadmium intoxication (15-17). In particular, polyphenols are involved in regulating several chronic diseases as a result of their antioxidant properties, induction of detoxification enzymes, inhibition of enzymes, antiproliferative activity, control of cell cycle and modulation of apoptosis (18).

Grapes are fruits commonly consumed around the world and contain significant amounts of phenolic compounds (19). The chemical characterization of grape skin extract revealed the presence of many bioactive compounds, such as phenolic acids, flavonoids, anthocyanins and resveratrol. According to Vauzour et al. (20), polyphenols are chemopreventive agents by means of several biological mechanisms, such as removal 

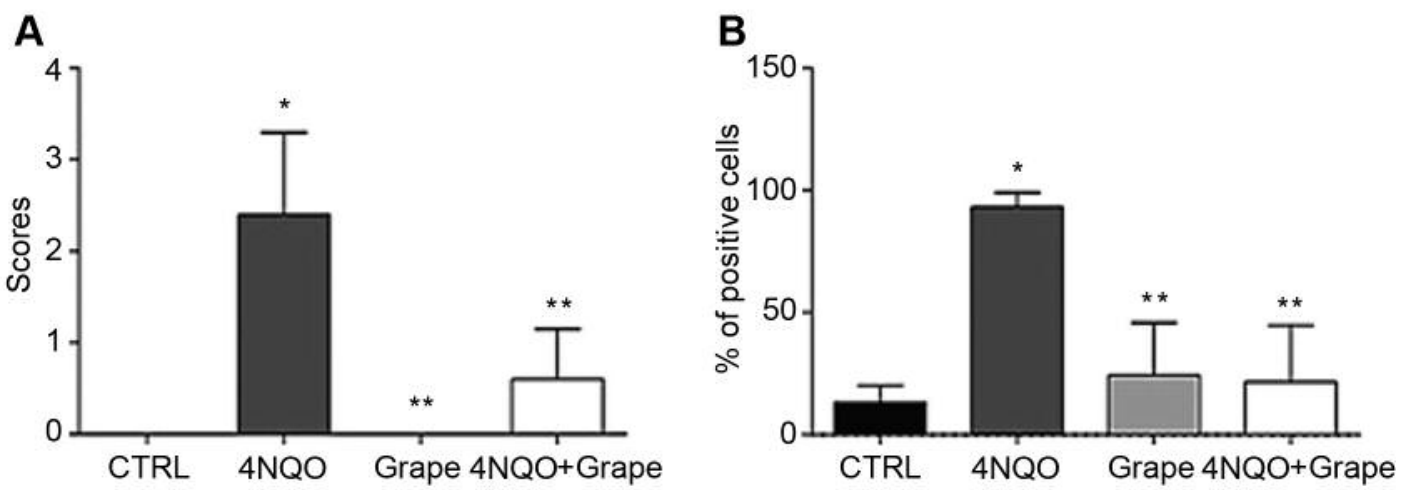

Figure 1. Immunohistochemical data for Ki-67 (A) and 8-OHdG $(B)$ in rat tongue mucosa following induction of oral carcinogenesis and treatment with or without grape skin extract. ${ }^{*} p<0.05$ compared to control group. ${ }^{* *} p<0.05$ compared to $4 N Q O$ group.
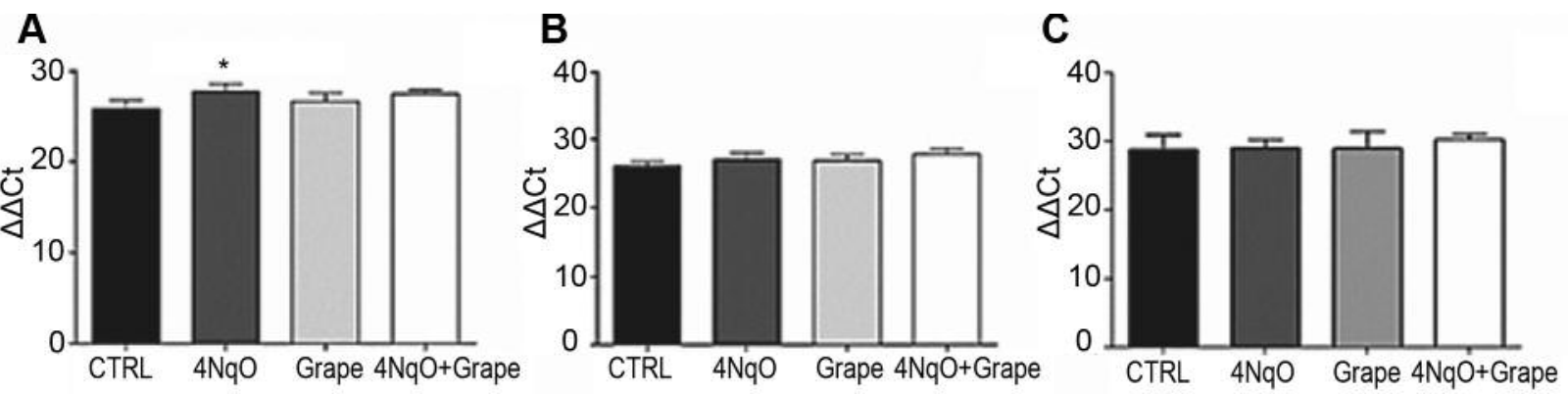

Figure 2. Expression of genes encoding antioxidant enzymes evaluated by Real-Time PCR. A: CuZn-SOD gene expression; B: Mn-SOD gene expression and $C$ : calatase gene expression. ${ }^{*} p<0.05$ when compared to control group.

of carcinogens, modulation of cancer cell signaling proteins and cell cycle arrest, induction of apoptosis and modulation of enzymatic activities. Recently, Gollücke and colleagues (21) have also shown that grape skin extract was effective against cadmium intoxication in rats since the extract was able to promote tissue regeneration and displayed antigenotoxic and anti-oxidant activities in rat liver cells.

Proanthocyanidin derived from grapes suppressed cellular proliferation and induced apoptosis in human squamous cell carcinoma cells in vitro (22). Lin and coworkers (23) described that procyanidins have an inhibitory effect on oral squamous cell carcinoma cells growth and invasion or metastasis as a result of apoptosis induction. Resveratrol, one of the most studied polyphenols present in grapes, had also inhibitory effects on growth and proliferation in and oral squamous cell line (24). Our results are fully in line with the above reports, since this study showed that grape skin extract was able to decrease the occurrence of epithelial dysplasia and decreased ki-67 immunostaining in rat tongue cells following $4 \mathrm{NQO}$ exposure.
With regard to antioxidant activity, it seems that grape polyphenols also reduce oxidative stress by scavenging a wide range of reactive oxygen species (ROS) and/or upregulating the antioxidant defenses (25). Previous studies have demonstrated that grape juice reduced the oxidative stress induced by $8-\mathrm{OHdG}$, by modulating endogenous antioxidant enzymes (15). Our results demonstrated that grape skin extract decreased expression of $8-\mathrm{OHdG}$ in rat tongue cells following chemical carcinogenesis.

Endogenous antioxidants enzymes, such as SOD and catalase, act to neutralize free radicals and protect the body against tissue damage (26). CuZn-SOD functions in the cytosol and its activity is not directly affected by oxidative stress whereas Mn-SOD activity resides in mitochondria, and is increased with oxidative stress (26). Catalase is responsible for converting hydrogen peroxide into water (26). Our data showed that CuZn-SOD was increased in the group that received $4 \mathrm{NQO}$ only. This result is consistent with that of others (27). It is important to stress that animals treated with grape skin extract did not alter 


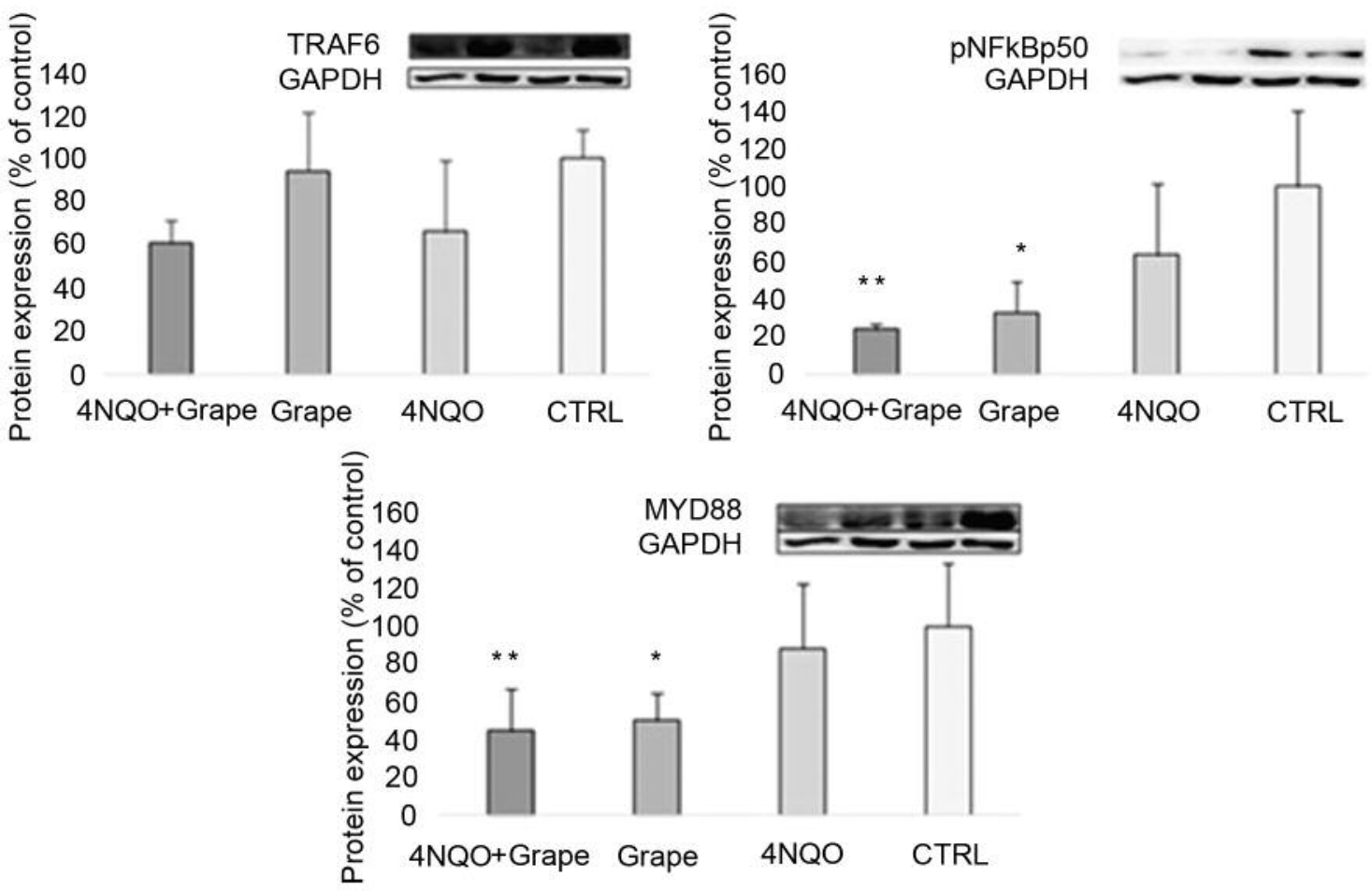

Figure 3. Western blot analysis of TRAF6, pNFkBp50 and MYD88 expression following induction of rat tongue carcinogenesis and treatment with grape skin extract. Data are expressed as Mean $\pm S . D . * p<0.05$ compared to control group; $* * p<0.05$ compared to 4 NQO group.

the levels of antioxidant enzymes, SOD-CuZn, SOD-Mn or catalase. Since grape skin extract is an antioxidant agent, it is plausible that the antioxidants enzymes not to be affected after $4 \mathrm{NQO}$ exposure. Therefore, it is assumed that grape skin extract is a potent antioxidant agent able to prevent oxidative DNA damage in rat mucosa cells. This biological action plays a crucial role either in the initiating or in the promoting phases of chemical carcinogenesis.

Accumulating evidence suggests that NF-kB pathway plays an important role in the promoting phase of carcinogenesis by regulating the immune function, inflammation, apoptosis, proliferation and stress responses, as well as the progression and invasion of cancer cells (28). Most frequently, activation of toll-like receptors leads to activation of NF-kB (TLR) (29). This occurs via myeloid differentiation primary response gene (MyD88), a common signal adaptor molecule of TLRs (29). In this study, the levels of p-NFKB p50 and MyD 88 decreased in animals treated with grape skin extract. Such findings lead us to postulate that grape skin extract possesses an antiinflammatory effect via the TLR signaling pathway.

In summary, our results indicate that grape skin extract is a chemopreventive agent against rat tongue carcinogenesis as a result of its anti-oxidant, anti-proliferative and antiinflammatory properties.

\section{Conflicts of Interest}

All Authors declare that no conflict of interest exists regarding this study.

\section{Acknowledgements}

CFGM and GRS are recipients from CAPES (Coordination of Superior Level Staff Improvement, Brazil) fellowships. WV, LPP and DAR are recipients of CNPq (National Council for Scientific and Technological Development, Brazil) fellowships. MJDS and ABS are recipients from FAPESP (Sao Paulo Research Foundation, Brazil) fellowships.

\section{References}

1 Ferlay J, Soerjomataram I, Ervik M, Dikshit R, Eser S, Mathers C, Rebelo M, Parkin DM, Forman D and Bray F: GLOBOCAN 2012 v1.0, Cancer Incidence and Mortality Worldwide: IARC CancerBase No. 11 [Internet]. Lyon, France: International Agency for Research on Cancer; 2013. Available from: http://globocan.iarc.fr

2 Thuler L and Rebelo M: Epidemiologia do câncer. 1st ed. Kowalsky L, Dib L, Ikeda M and Adde C (eds.). Frôntis [in Portuguese], 1999.

3 Hahn M, Hagedorn G, Kuhlisch E, Schackert H and Edkelt U: Genetic polymorphisms of drug-metabolizing enzymes and susceptibility to oral cavity cancer. Oral Oncol 38: 486-490, 2002. 
4 Ribeiro DA, Fávero Salvadori DM, da Silva RN, Ribeiro Darros $B$ and Alencar Marques ME: Genomic instability in nonneoplastic oral mucosa cells can predict risk during 4nitroquinoline 1-oxide-induced rat tongue carcinogenesis. Oral Oncol 40(9): 910-915, 2004.

5 Miranda S, Noguti J, Carvalho J, Oshima C and Ribeiro D: Oxidative DNA damage is a preliminary step during rat tongue carcinogenesis induced by 4-nitroquinoline 1-oxide. J Mol Histol 42(2): 181-186, 2011.

6 Fracalossi A, Miranda S, Oshima C, Franco M and Ribeiro D: The role of matrix metalloproteinases 2 and 9 during rat tongue carcinogenesis induced by 4-nitroquinoline 1-oxide. J Mol Histol 41: 19-25, 2010.

7 Fracalossi A, Silva M, Oshima C and Ribeiro D: Wnt/ $\beta$-catenin signalling pathway following rat tongue carcinogenesis induced by 4-nitroquinoline 1-oxide. Exp Mol Patol 88: 176-183, 2010.

8 Ribeiro DA, Kitakawa D, Domingues MA, Cabral LA, Marques ME and Salvadori DM: Survivin and inducible nitric oxide synthase production during 4-NQO-induced rat tongue carcinogenesis: a possible relationship. Exp Mol Pathol 40: 307313, 2007.

9 Ribeiro FA, Peres RC, Oshima CT, Spolidorio LC, Maluf Lle S and Ribeiro DA: Antioxidant activity of apple extract protects against rat tongue carcinogenesis induced by 4-nitroquinoline 1oxide. Toxicol Mech Methods 25(7): 532-537, 2015.

10 Georgiev V, Ananga A and Tsolova V: Recent advances and uses of grape flavonoids as nutraceuticals. Nutrients 6(1): 391-415, 2014.

11 Gollucke AP, Peres RC, Odair A Jr. and Ribeiro DA: Polyphenols: a nutraceutical approach against diseases. Recent Pat Food Nutr Agric 5(3): 214-219, 2013.

12 De Jesus GP, Ribeiro FA, de Moura CF, Gollucke AP, Oshima CT and Ribeiro DA: Anti-tumor activity of grape juice concentrate in the rat tongue two-stage initiation-promotion protocol induced by 4-nitroquinoline 1-oxide. Toxicol Mech Methods 24(4): 276-283, 2014.

13 Hasler CM, Bloch AS, Thomson CA, ENrione E and Manning C: Position of the American Dietetic Association: Functional Foods. J Am Diet Associ 104(5): 814-826, 2004.

14 Yujra VQ, Antunes HKM, Mônico-Neto M, Pisani LP, Santamarina AB, Quintana HT, de Oliveira F, Oshima CTF and Ribeiro DA: Sleep deprivation induces pathological changes in rat masticatory muscles: Role of Toll like signaling pathway and atrophy. J Cell Biochem 119(2): 2269-2277, 2018.

15 De Moura CF, Ribeiro FAP, De Jesus GP, Da Silva VH, Oshima CT, Gollücke AP, Aguiar O Jr and Ribeiro DA: Antimutagenic and antigenotoxic potential of grape juice concentrate in blood and liver of rats exposed to cadmium. Environ Sci Pollut Res Int 21(22): 13118-1326, 2014.

16 Da Silva AO, Gollücke APB, Noguti J, Yamamura ETH and Ribeiro DA: Grape juice concentrate modulates p16 expression in high fat diet-induced liver steatosis in Wistar rats. Toxicol Mech Methods 22(3): 218-224, 2012.
17 Paiotti AP, Neto RA, Marchi P, Silva RM, Pazine VL, Noguti J, Pastrelo MM,Gollücke AP, Miszputen SJ and Ribeiro DA: The anti-inflammatory potential of phenolic compounds in grape juice concentrate $\left(\mathrm{G} 8000^{\mathrm{TM}}\right)$ on 2,4,6-trinitrobenzene sulphonic acid-induced colitis. Br J Nutr 110(6): 973-980, 2013.

18 Upadhyay S and Dixit M: Role of polyphenols and other phytochemicals on molecular signaling. Oxid Med Cell Longev 2015: 504253, 2015.

19 Hasan $\mathrm{M}$ and Bae H: An Overview of stress-induced resveratrol synthesis in grapes: perspectives for resveratrol-enriched grape products. Molecule 22(2): pii: E294, 2017.

20 Vauzour D, Rodriguez-Mateos A, Corona G, Oruna-Concha MJ and Spencer JP: Polyphenols and human health: prevention of disease and mechanisms of action. Nutrients 2(11): 1106-1031, 2010.

21 Gollucke APB, Claudio SR, Yamamura H, Morais DR, Bataglion GA, Eberlin MN, Aguiar O Jr and Ribeiro DA: Grape skin extract mitigates tissue degeneration, genotoxicity, and oxidative status in multiple organs of rats exposed to cadmium. Eur $\mathbf{J}$ Cancer Prev 27(1): 70-81, 2018.

22 King M, Chatelain K, Farris D, Jensen D, Pickup J, Swapp A, O'Malley S and Kingsley K: Oral squamous cell carcinoma proliferative phenotype is modulated by proanthocyanidins: a potential prevention and treatment alternative for oral cancer. BMC Complement Altern Med 7: 22, 2007.

23 Lin YS, Chen SF, Liu CL and Nieh S: The chemoadjuvant potential of grape seed procyanidins on p53-related cell death in oral cancer cells. J Oral Pathol Med 41(4): 322-331, 2012.

24 El Attar TM and Virji AS: Modulating effect of resveratrol and quercetin on oral cancer cell growth and proliferation. Anticancer Drugs 10(2): 187-193, 1999.

25 Sen S, Chakraborty R, Sridhar C, Reddy YSR and De B: Free radicals, antioxidants, diseases and phytomedicines: current status and future prospect. Int J Pharm Sci Rev Res 3(1): 91-100, 2010.

26 Pisoschi AM and Pop A: The role of antioxidants in the chemistry of oxidative stress: A review. Eur J Med Chem 97: 5574,2015 .

27 Bishayee A, Ahmed S, Brankov N and Perloff M: Triterpenoids as potential agents for the chemoprevention and therapy of breast cancer. Front Biosci (Landmark Ed) 16: 980-996, 2011.

28 Mitjavila MT and Moreno JJ: The effects of polyphenols on oxidative stress and the arachidonic acid cascade. Implications for the prevention/treatment of high prevalence diseases. Biochem Pharmacol 84(9): 1113-1122, 2012.

29 Pisani LP, Estadella D and Ribeiro DA: The role of toll like receptors (TLRs) in oral carcinogenesis. Anticancer Res 37(10): 5389-5394, 2017. 